\title{
Cloning and characterization of a mannose binding C-type lectin gene from salivary gland of Aedes albopictus
}

\author{
Jinzhi Cheng ${ }^{1,2}$, Yu Wang ${ }^{1,3}$, Fangzhan Li ${ }^{1,2}$, Jian Liu' ${ }^{1,2}$, Yu Sun ${ }^{1,4}$ and Jiahong $\mathrm{Wu}^{1,2^{*}}$
}

\begin{abstract}
Background: The studies on sialomes have shown that hematophagous mosquito saliva consists of a lot of pharmacologically active proteins, in which C-type lectins have been identified and regarded as an important component of saliva. The previous studies showed that C-type lectins play crucial roles not only in innate immunity but also in promoting disease transmission in mammals. However, the function and mechanism of C-type lectins from the mosquito sialome is still elusive.

Methods: A putative C-type lectin gene (Aalb_CTL1) was cloned and expressed from Aedes albopictus by RT-PCR. The deduced amino acid sequence was analyzed by bioinformatic methods. The gene expression profiles in different tissues and various blood-fed stages of Ae. albopictus were examined by Real-Time qRT-PCR and the biological functions of the recombined mature Aalb_CTL1 were tested by hemagglutination and sugar inhibitory agglutination assays. Moreover, the capabilities of rAalb_CTL1 against microorganisms were measured by microbial-agglutination assay.

Results: The full-length Open reading frame (ORF) of Aalb_CTL1 consisted of 462 bp, encoding 153 amino acid residues. The deduced amino acid sequence contained a putative signal peptide of 19 amino acids. It also contained a CRD domain with a WND (Trp ${ }^{137}-$ Asn $^{138}-$ Asp- $^{139}$ ) motif that needed calcium for the hemagglutinating activity and an imperfect EPS (Glu ${ }^{128}-$ Pro $^{129}-$ Ser $\left.^{130}\right)$ motif that had a predicted ligand binding specificity for mannose. The mRNA level of Aalb_CTL1 was much higher in female mosquito salivary gland than those in fat body and midgut which was down-regulated in salivary gland after blood feeding. The rAalb_CTL1 contained not only hemagglutinating activity and a high affinity with mannose but also agglutinating activity against yeast C. albicans and Gram-positive bacteria S. aureus in $\mathrm{Ca}^{2+}$ dependent manner.
\end{abstract}

Conclusion: Aalb_CTL1 was a mannose-binding C-type lectin and constituted one of the important components in saliva of Ae. albopictus, which could be involved in the defense against yeast and Gram-positive bacteria infection.

Keywords: C-type lectin, Salivary gland, Ae. albopictus, Prokaryotic expression, Agglutinating activity

\section{Background}

C-type lectins (CTLs) constitute the largest and most diverse super family of proteins that share a characteristic carbohydrate recognition domain (CRD) with two or three pairs of disulfide bonds [1,2]. Due to their ability to bind to specific carbohydrates on the surface of

\footnotetext{
* Correspondence: jiahongwu2008@hotmail.com

'Department of Parasitology, Guiyang Medical College, Guiyang, Guizhou

550004, China

${ }^{2}$ Lab for Modern Pathogen Biology, Guiyang Medical College, Guiyang,

Guizhou 550004, China

Full list of author information is available at the end of the article
}

microorganisms, CTLs have been regarded as primary candidates for pattern recognition receptors (PRRs) to mediate pathogen recognition, which play an important role in the clearance of pathogens in innate immunity in vertebrates [3,4]. In invertebrates, the previous studies demonstrated that CTLs are also involved in innate immune responses, including the promotion of phagocytosis [5], nodule formation, encapsulation, melanization [6,7] and the activation of prophenoloxidase [8].

Mosquitoes are one of the most important medical arthropods. The harm to human beings caused by mosquitoes 
are not only in its harassment and blood-feeding habits, but also in its transmission of various diseases, such as malaria, dengue fever, Japanese encephalitis, yellow fever, Venezuelan equine encephalitis and Ross River fever [9]. The whole genomics studies demonstrated that there are 25, 39 and 55 CTLs respectively in Anopheles gambiae [10], Aedes aegypti [11] and Culex quinquefasciatus [12], which mostly are soluble proteins containing a single CRD without accessory domains. Several CTLs have exhibited a diverse range of function in mosquito innate immunity. The CTL4 and CTLMA2 from malaria vector $A n$. gambiae can inhibit Plasmodium berghei ookinete melanization, indicating that they act as agonists of Plasmodium development in the vector [13]. In Ae. aegypti, a C-type lectin ( AaegmosGCTL-1 ) induced by West Nile Virus (WNV) infection, can interact with WNV in a calciumdependent manner, and facilitate flavivirus invasion in vivo and in vitro [14]. By in vivo reverse genetic analysis, 9 mosGCTL genes from Ae. aegypti are identified as important susceptible factors to produce DENV-2 infection, of which mosGCTL-3 exhibits the most significant effect [15]. Based on the evidence above, it can be suggested that mosquito CTLs could be used as ligands by pathogens to promote the infection of a vector. On the other hand, the CTL4 and CTLMA2 have also been found to be required for the clearance of Escherichia coli, but not Staphylococcus aureus, suggesting that they play a role in fighting against Gram-negative bacteria infection [16]. Therefore, it could be inferred that the CTLs from mosquitoes could serve pleiotropic functions in the interaction of mosquito and pathogen.

Ae. albopictus is an efficient laboratory vector for a large number of arboviruses such as dengue virus, yellow fever virus, West Nile virus and several others. In China, it is also an important vector to transmit dengue fever. In Ae albopictus, two CTLs, AY826070 and AY806069, have been identified by sialotranscriptome analysis [17]. In this study, the open reading frame (ORF) of AY826070 from Ae. albopictus Guangzhou strain (named Aalb_ CTL1) was cloned by RT-PCR and expressed with prokaryotic expression system. The results of Real-Time quantitative RT-PCR (Real-Time qRT-PCR) showed that Aalb_CTL1 was expressed specifically in female mosquito salivary gland. rAalb_CTL1 demonstrated agglutinating activity against animal erythrocyte, Gram-positive bacteria and yeast. It laid a preliminary foundation to make further study on the role of Aalb_CTL1 in salivary immunity.

\section{Methods}

\section{Mosquitoes and sample collection}

Ae. albopictus mosquitoes (Guangzhou strain) were kindly provided by Prof. Zhao Tongyan from Beijing Institute of Microbiology and Epidemiology and routinely reared at $25 \pm 1^{\circ} \mathrm{C}$ with $75-80 \%$ relative humidity and a 14:10 (light: dark) photoperiod. Mosquitoes were fed on anesthetized mice.

Tissue samples including salivary glands, midgut and fat body from the sugar-fed adult female mosquitoes at 3-5 days old were dissected in the normal saline (NS: $0.9 \% \mathrm{NaCl}, \mathrm{pH} 6.5)$ and immediately soaked in the TRIzol Reagent (Invitrogen Life Technologies) and stored at $-80^{\circ} \mathrm{C}$ until use. Six biological replicates, each consisting of salivary glands from 6 mosquitoes, midgut and fat body from 1 mosquito, were collected for each sample.

Salivary glands were further dissected at various time points: $3-5$ days post emergence (PE) and $0 \mathrm{~h}, 24 \mathrm{~h}$ and $72 \mathrm{~h}$ post blood-feeding. Mosquitoes, 3-5 days PE, were fed on anesthetized mice for $2 \mathrm{~h}$, and the mosquitoes with engorged abdomen were selected and regarded as $0 \mathrm{~h}$ post blood-feeding. Six biological replicates, each consisting of salivary glands from 6 mosquitoes, were collected for each time point.

\section{Full-length ORF cloning of Aalb_CTL1}

Total RNA was extracted from female mosquito with TRIzol Reagent according to the manufacturer's protocol. The single-stranded cDNA was synthesized using PrimeScript $^{\text {Tim }}$ RT Kit (TaKaRa). Aalb_CTL1 specific primers for full length ORF (Table 1) were designed according to the published cDNA sequence of Ae. albopictus [GenBank: AY826070]. The desired products were amplified by 35 cycles of PCR using $2 \mu$ synthesized cDNA as template in $50 \mu \mathrm{l}$ reaction containing $5 \mu \mathrm{l} 10 \times \mathrm{Ex}$ Taq buffer (containing $\mathrm{Mg}^{2+}$ ), 40pmol each primer and $5 \mathrm{U} \mathrm{Ex}$ Taq DNA Polymerase (TaKaRa). The PCR conditions were $5 \mathrm{~min}$ at $94^{\circ} \mathrm{C}$ for one cycle, followed by 35 cycles of denaturation at $94^{\circ} \mathrm{C}$ for $30 \mathrm{sec}$, annealing at $58^{\circ} \mathrm{C}$ for $30 \mathrm{sec}$, and extension at $72^{\circ} \mathrm{C}$ for $1 \mathrm{~min}$. In the last cycle, the PCR product was further incubated at $72^{\circ} \mathrm{C}$ for $7 \mathrm{~min}$ to allow the completion of DNA synthesis.

The amplified products were purified using DNA purification kit (TaKaRa). The purified PCR products were cloned into the pMD18-T cloning vector $(\mathrm{TaKaRa})$ at mole ratio $5: 1$ at $16^{\circ} \mathrm{C}$ and then transformed into freshly prepared E. coli strain DH5a competent cells. Recombinant clones were selected using blue/white screening on X-gal $(25 \mu \mathrm{g} / \mathrm{mg}) / \mathrm{IPTG}(25 \mu \mathrm{g} / \mathrm{ml}) /$ Ampicillin $(50 \mu \mathrm{g} / \mathrm{ml})$ LB plates. The clones were confirmed by Nde I and Hind III restriction enzymes digestion. Three positive clones were picked up for sequencing in both directions.

\section{Sequences analysis}

The homology analyses of the nucleotides and deduced amino acid sequences of Aalb_CTL1 were carried out using the BLAST programs at the NCBI (http://www. ncbi.nlm.nih.gov/blast). Translation of the cDNA was performed using the Expert Protein Analysis System 
Table 1 Primers used in present study

\begin{tabular}{llll}
\hline Gene name & Forward primer (5'-3') & Reverse primer (5'-3') & Amplicon size (bp) \\
\hline $\begin{array}{l}\text { Aalb_CTL1 ORF cloning } \\
\text { Aalb_CTL1 } \quad \text { ATGGGGCAGCCATCATCATCATCAT }\end{array}$ & ACATTCGTTCGCACACAAAAC & 462 \\
$\begin{array}{l}\text { Recombinant expression } \\
\text { Aalb_CTL1 }\end{array}$ GTCCATATGCAACAGGAATGCGACTGTAAAAATAG & GTGAAGCTTACATTCGTTCGCACACAAAAC \\
$\begin{array}{l}\text { Quantitative real-time PCR (Q-PCR) } \\
\text { Aalb_CTL1 }\end{array}$ GCTATCTCTCTCGCAGACCTCAC & CCAATCTCCAGCCGTTCCT & 405 \\
Rsp 5 & ATTACATCGCCGTCAAGGAG & TCATCATCAGCGAGTTGTC & 130 \\
\hline
\end{tabular}

The restriction endonuclease sites in italics.

(http://au.expasy.org). Signal peptide and CRD domain predictions were conducted using SMART software (http://smart.embl-heidelberg.de/). The homologous sequences from various species were downloaded from NCBI. A phylogenetic tree was constructed using the Neighbour-joining method with the Molecular Evolutionary Genetics Analysis (MEGA 4) package.

Real-Time quantitative Reverse Transcription Polymerase Chain Reaction (Real-Time qRT-PCR) analysis.

Total RNA was extracted for all the tissue samples separately with TRIzol Reagent according to the manufacturer's protocol. Before reverse transcription, the RNA samples were treated with DNAase I (Invitrogen) and the single-stranded cDNAs were synthesized using PrimeScript $^{\text {Ti }}$ RT Kit (TaKaRa). According to the cDNA full-length sequence, one pair of gene-specific primers for Aalb_CTL1 (Table 1) was used to amplify product of $130 \mathrm{bp}$ from the cDNA. Two rsp5 primers (Table 1) were used to amplify a $126 \mathrm{bp}$ fragment as an internal control to verify the successful transcription and to calibrate the cDNA template for corresponding mosquito samples [18].

Real-Time PCR amplification was carried out in an ABI 7300 Real-Time Thermal Cycler according to the manual (TaKaRa). Dissociation curve analysis of amplification products was performed at the end of each PCR to confirm that only one PCR product was amplified and detected. The two/double standard curve method was used to analyze the expression level of Aalb_CTL1. All data were given in terms of mRNA expressed as means \pm SE. Significant differences between means were determined using one way ANOVA and $\mathrm{P}<0.05$ was considered statistically significant.

\section{Prokaryotic expression and purification of recombinant Aalb_CTL1}

A cDNA fragment encoding a mature Aalb_CTL1 protein (residues 20 to 153) was amplified by PCR using Ex Taq polymerase (TaKaRa) with the specific primers for recombinant expression (Table 1). Nde I and Hind III restriction sites were added to the $5^{\prime}$ ends of Aalb_CTL1 primers (after the stop codon), respectively. The PCR fragment was cloned into the pMD18-T vector (TaRaKa) firstly, it was completely digested with Nde I and Hind III (TaRaKa), and cloned into the Nde I and Hind III sites of the expression vector pET28a $(+)$ subsequently, then the recombinant plasmid (pET28-Aalb_CTL1) was transformed into E. coli BL21 (DE3). Positive clones were screened by enzymatic digestion with Nde I and Hind III and confirmed by nucleotide sequencing. Finally, positive transformants were incubated in $100 \mathrm{ml}$ LB medium (10 g tryptone, $5 \mathrm{~g}$ yeast extract, $10 \mathrm{~g} \mathrm{NaCl} / 1 \mathrm{l}$ distilled water, $\mathrm{pH} 7.0$ ) at $37^{\circ} \mathrm{C}$ with shaking at $220 \mathrm{rpm}$ until the culture reached OD600 of 0.5-0.7, and $1 \mathrm{mmol} / \mathrm{L}$ isopropyl- $\beta$-D-thiogalactosidase (IPTG) was added to the medium under the same conditions for another $5 \mathrm{~h}$. Bacterial cells expressing recombinant proteins were harvested and sonicated, and the inclusion bodies were resuspended in $1 \times$ phosphate-buffered saline (PBS: $\mathrm{NaCl}$ $137 \mathrm{mmol} / \mathrm{L}, \mathrm{KCl} 2.7 \mathrm{mmol} / \mathrm{L}, \mathrm{Na}_{2} \mathrm{HPO}_{4} 10 \mathrm{mmol} / \mathrm{L}$, $\mathrm{KH}_{2} \mathrm{PO}_{4} 2 \mathrm{mmol} / \mathrm{L}, \mathrm{pH}$ 7.4) containing $8 \mathrm{M}$ urea. Recombinant Aalb_CTL1 protein (rAalb_CTL1) was purified by gradient dialysis at $4^{\circ} \mathrm{C}$ overnight, and further purified with His•Bind Purification Kit (Novagen) according to the manufacturer's instructions. Purified recombinant protein was dialyzed against $1 \times \mathrm{PBS}$ buffer at $4^{\circ} \mathrm{C}$ overnight. The resulting protein was analyzed by $15 \%$ sodium dodecyl sulfate-polyacrylamide gel electrophoresis (SDS-PAGE) and visualized with Coomassie brilliant blue R-250.

\section{Hemagglutination assays}

To test whether or not calcium was required for the hemagglutinating activity of rAalb_CTL1, $12.5 \mu \mathrm{l}$ of serial dilutions of $\mathrm{CaCl}_{2}$ in NS was mixed with $12.5 \mu \mathrm{l}$ of rAalb_CTL1 $(\sim 50 \mu \mathrm{g} / \mathrm{ml})$. BSA instead of Aalb_CTL1 was used as a negative control. After $25 \mu \mathrm{l} 2 \%$ suspension of mouse erythrocytes was added, the mixture was incubated for $30 \mathrm{~min}$ at $25^{\circ} \mathrm{C}$. Hemagglutination was observed under a microscope (Nikon TE2000, Japan). Assays were performed in triplicate independently.

The hemagglutinating activity of rAalb_CTL1 was tested using $2 \%$ (vol/vol) erythrocytes from rabbits, mice, and rats according to the method described by Luo et al. [19]. Erythrocytes were washed five times with NS-Ca 
buffer $(0.9 \% \mathrm{NaCl}, 40 \mathrm{mM} \mathrm{CaCl} 2, \mathrm{pH}$ 6.5) and then diluted with NS-Ca buffer to $2 \%$ (vol/vol) suspension. Two-fold serial dilutions $(25 \mu \mathrm{l})$ of rAalb_CTL1 in NS-Ca buffer were mixed with $25 \mu \mathrm{l}$ of the erythrocyte suspension in a microtiter $\mathrm{V}$ plate. The plate was incubated for $1 \mathrm{~h}$ at room temperature, and the hemagglutination was observed under a microscope (Nikon TE2000, Japan). Erythrocyte suspension mixed with serial dilutions of bovine serum albumin (BSA, Sigma) in NS-Ca buffer were processed in parallel to serve as negative controls.

\section{Sugar binding specificity of rAalb_CTL1}

The sugar binding specificity of rAalb_CTL1 was determined by an inhibitory agglutination assay. Serial dilutions $(12.5 \mu \mathrm{l})$ of various carbohydrates (Sigma) in NS-Ca buffer, including D-mannose, D-galactose, Lactose, Dglucose, Maltose and Sucrose, were mixed with $12.5 \mu \mathrm{l}$ of rAalb_CTL1 $(12 \mu \mathrm{g} / \mathrm{ml})$ and incubated for $30 \mathrm{~min}$ at $37^{\circ} \mathrm{C}$. Then, $2 \%$ erythrocytes suspension was added, and the mixture was incubated for $30 \mathrm{~min}$ at room temperature. An inhibitory effect was observed at the minimum concentration of a carbohydrate required for complete inhibition of the hemagglutinating activity of rAalb_CTL1. This assay was performed in triplicate independently.

\section{Microbial-agglutination assays}

Three microorganisms were used for agglutination tests including yeast C. albicans (ATCC10231), Gram-negative bacteria E. coli (ATCC25922) and Gram-positive bacteria S. aureus (ATCC25923). All three microorganisms were obtained from Beijing Institute of Microbiology and Epidemiology and maintained in our laboratory. Bacteria were streaked on LB agar plates at $37^{\circ} \mathrm{C}$ for $18 \mathrm{~h}$ firstly, then a single colony was inoculated into $5 \mathrm{ml} \mathrm{LB}$ medium and cultured overnight at $37^{\circ} \mathrm{C}$. The bacteria were cultured again in fresh medium at 1:100 dilution for $5 \mathrm{~h}$ at $37^{\circ} \mathrm{C}$ and collected in mid logarithmic phase by centrifugation at $6000 \mathrm{rpm}$ for $5 \mathrm{~min}$.

C. albicans were streaked in Sabouraud's agar plate (10 g tryptone, $40 \mathrm{~g}$ glucose $/ 1 \mathrm{l}$ distilled water, $\mathrm{pH}$ 5.6; with $20 \mathrm{~g}$ agar) at $25^{\circ} \mathrm{C}$ for $24 \mathrm{~h}$, then a single colony was inoculated into $5 \mathrm{ml}$ Sabouraud's medium and cultured for $36 \mathrm{~h}$ at $25^{\circ} \mathrm{C}$. The yeast was cultured again in fresh Sabouraud's medium at 1:100 dilution for $24 \mathrm{~h}$ at $25^{\circ} \mathrm{C}$ and collected by centrifugation at $6000 \mathrm{rpm}$ for 5 min.

The yeast or the bacteria prepared as above were stained by DAPI for $20 \mathrm{~min}$, washed twice with TBS buffer (Tris $50 \mathrm{mmol} / \mathrm{L}, \mathrm{NaCl} 150 \mathrm{mmol} / \mathrm{L}, \mathrm{pH} 7.6$ ) and resuspended in TBS buffer at a concentration of $2 \times 10^{8}$ cells $/ \mathrm{ml}$. Then $50 \mu \mathrm{l}$ TBS including rAalb_CTL1 at final concentration $50 \mu \mathrm{g} / \mathrm{ml}$ was added into $50 \mu \mathrm{l}$ of bacteria/yeast. After incubation for $1 \mathrm{~h}$ at room temperature in the presence or absence of $40 \mathrm{mM} \mathrm{CaCl}$, these samples were observed and photographed under a fluorescence microscope (Nikon TE2000, Japan). BSA instead of rAalb_CTL1 was used as a negative control (BSA-Ca control).

\section{Ethical approval}

Ethical approval documents were given by the Experimental Animal Ethics Committee of Guiyang Medical College.

\section{Results and discussion}

Saliva of mosquitoes consists of a milieu of pharmacologically active substances to function not only in food uptake but also in disease transmission [20]. Sialome studies have shown that there are at least 100 proteins in mosquito saliva, and more than one CTL has been identified and predicted in mosquito sialomes [17,21,22]. However, little is known about the functions of them. In our study, we detected the agglutinating activity of Aalb_CTL1.

\section{CDNA cloning and sequence analysis of Aalb_CTL1}

Based on the cDNA sequence of Aalb_CTL1, gene-specific primers were designed and the ORF of Aalb_CTL1 was cloned and sequenced from the Ae. albopictus Guangzhou strain. A total of $462 \mathrm{bp}$ encoding a protein of 153 amino acids was obtained (Figure 1A). Sequence alignment showed there was a base mutation at position 160 from A to $G$, which in turn caused an amino acid change from I to $\mathrm{V}$ in the Ae. albopictus Guangzhou strain. Multiple sequence alignment of carbohydrate recognition domain (CRDs) from Ae.aegypti and Cx. quinquefasciatus demonstrated that $\mathrm{V}$ was more conservative than $\mathrm{I}$ in this position (Figure 1B). It is well known that both $\mathrm{V}$ and I belong to the aliphatic amino acids, but a further study would be performed to test the effect on function of Aalb_CTL1 with such an amino acid change.

According to the ORF of Aalb_CTL1, the deduced amino acids sequence contained a putative signal peptide of 19 residues at the $\mathrm{N}$-terminal, suggesting that it should be a secreted protein and would be a component of saliva in mosquito. The calculated molecular mass of the mature Aalb_CTL1 protein (residues 19 to 153 ) is $15.71 \mathrm{kDa}$, with an estimated pI of 5.72. Like other C-type lectins, Aalb_CTL1 had a single characteristic CRD stabilized by two highly conserved disulphide bridges from four cysteine residues Cys 45, Cys 122, Cys 142 and Cys 150 [23] (Figure 1A). Multiple sequence alignment of CRDs (Figure 1B) demonstrated that the CRD of Aalb_CTL1 contained 13 of 14 invariant and 14 of 18 highly conserved amino acid residues, which were identified as a C-type CRD by Drickamer et al. [23] and Yamanoto-Kihara et al. [24]. A WND motif (Trp137-Asp138-Asn139) was identified in the C-terminal of CRD of Aalb_CTL1, indicating 

A
1 ATGATTTTCTCATTG TATCTAATCGTGGCT ATCTCTCTCGCAGAC CTCACAGCCGCCCAA $\begin{array}{llllllllllllllllllll}I I & I & F & S & L & Y & L & I & V & A & I & S & L & A & D & L & T & A & A & Q\end{array}$
61 CAGGAATGCGACTGT AAAAATAGATTCTGC TTCCCAAACGTTGTG GCAAACTGGATCGGA

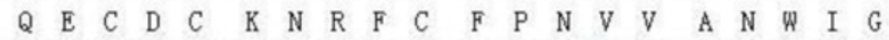
121 GCAGCCGAGTATTGC AGCAGGAACGGCTGG AGATTGGCCGTACTG GATACGGAGGAAAAG $\begin{array}{llllllllllllllllllll}A & A & E & Y & C & S & R & N & G & W & R & L & A & V & L & D & T & E & E & K\end{array}$
181 CAGCAACAGGTGGAG GAGCAGGCCCAGAAA GTGGACGCATTTAAA ACGAACAAGGTGGAG $\begin{array}{llllllllllllllllllll}Q & Q & Q & V & E & E & Q & A & Q & K & V & D & A & F & K & T & N & K & V & E\end{array}$
241 CTGTGGATCGGAGCC AGCGATTTGGCCAAG GAAGGCAAATTTGTT TGGCACGGTACCGGG $\begin{array}{lllllllllllllllllllll}L & W & I & G & A & S & D & L & A & K & E & G & K & F & V & \text { W } & H & G & T & G\end{array}$
301 ATTGATGTGTCGTAC GAGAAGTGGATCGCC GGCATGCCGGACAAT AGAAACGGTAATGAA $\begin{array}{llllllllllllllllllll}I & D & V & S & Y & E & K & \text { W } & \text { I } & A & G & \text { II } & P & D & N & R & N & G & N & E\end{array}$
361 CACTGCGTTCATCTG TGGTACGAACCGTCG CGTTCTTTCCAGTGG CAATGGAATGACGTG

 421 GTGTGCAGCTCAACG CGGCGTTTTGTGTGC GAACGAATGTAA
$\begin{array}{lllllllllllllll}V & C & S & S & T & R & R & F & V & C & E & R & \text { II } & *\end{array}$

\section{$\Omega \quad \Phi \quad \theta \quad \stackrel{*}{\mathbf{C}} \quad \theta \mathbf{\nabla} \theta 0 \mathrm{E} \quad \Omega \theta$}
Aalb_CTL1 (29) -EFCFNVANWIG IG AEYCSRNGWT AVIDTEEKQQQTEEQAQKVDAFK TN
(25) - YCFPNEVATW TG BVEYCLKN WKL AWNSFEAKQMK IEGLAKRLPEFKNG
(30) -FCFPNVANW IG ASEYCSRNG AVL DSEEKQQQVEELAQRVDAFKTA
(30) - FCFPNVANW IG AEYCSRNG WR AVD DEQKQQQ EELAQRVDAFKTA
(25) - FCFPNEVAT IG GVYCLRWG AWNSEAKQMK IESLAKNL GEFKRG
(28) -FYIPSIRAN FF ANEFCNSLKMIIL VAIRS QEDNDAVRRYVRTTSKF TDN
(29) - YLIPNFT ANV FK AVYCTYLGRFL ANIE P AAEENL YRAIK SSEVFVW
(51) L TTPNLRLNUFD WVYCSSIGMSIATIKD TNERQLLQLHLDGDRRL TRS
(46) YFIPISRTSD UFG GVAYCHSWGME AE ILNEDEARAMGEVIAE-EESDSD
Agan CTL
Agan CTLMA2

\section{$\Phi \in G \theta \quad \Phi \quad$ G $\quad \Omega \quad$ T $\quad$ P $\quad$ EO}

Aalb_CTL1

(78) K-D-VEL WIG ISDLAKEGKFVWHG TGIDVSWEKWIAG--MPPNRNGNEH

(74) K---VEL WIG ASDQTKEGHFW IANGQPIEVANYLPG--KPDNKDGKEH

Aalb_CTL2

Aaeg CTL20

Aaeg CTL16



(79) K-D-VEL UI G SDLAREGKFMWHP TGLDVSY SKWIAG--MPDNKDG YEH

Aaeg CTL25 (74) K---VELWIG SSDQTKEGNFV LANGKPVE ANWAIPG--KPDNKDGKEH

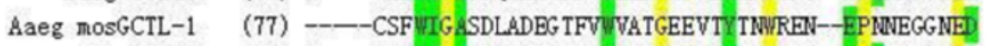

Cq CTL54 (78) S---TRVIIG GNDLGDEGNFHUHATGRVQYANWVHL--QPDNNWGGNEN

Ag an CTL4 (101) QKRSKIPYWIG\&NSLIAGQGLRIGLTDQEVKESAEWADGIAPANNRVPF

Ag an CTLMA2 (95) D---EFYWIGGNDLGWG TYR MAL TGRPV ॠSQWAAG-EPNHARG-EN

$$
\stackrel{*}{\mathrm{C}} \Omega \Omega \quad \quad \mathbf{W D} \stackrel{*}{\mathrm{C}} \quad \Omega \stackrel{*}{\mathrm{C}}
$$

Aalb_CTL1 (122) CYHHWYYPSR--SFQWQWNDWCSST-RRFWCERM-

Aalb_CTL2 (118) CWHLWYEKAK--KLWWGWNDWCTSK-RRFWCERKKK-

Aaeg CTL20 (123) CYH WYEPSR--LFNWHWNDWCASM-RRPWCEQA-

Aaeg CTL16 (123) CYHLWYPPSR--LINWHWNDWCASM-RRFWCEQA-

Aaeg CTL25 (118) CWH WYEKAK--NINYGWNWCTSK-RRFWCERKKN-----

Aaeg mosGCTL-1 (120) CIQLAYIPALN-YHWSUNDNTCAGQSLYYFICESVECDCWPF

Cq CTL54 (122) CYE GTNNWGFQLNWKWNDDCNRS-HYFACEEGS-----

Agan CTL4 (151) CYYMQGSTMS----WWATSCDDEPRQFICEY-

Agan CTLIMA2 (138) GQQPAERCVAVAMDKYEWNDFQCTQQ-KPFICQQFRKH---

Figure 1 Sequence analysis of Aalb_CTL1. (A) ORF and deduced amino acid sequence of Aalb_CTL1 from the Aedes albopictus Guangzhou strain. The amino acid sequence is represented by a single capital letter below the nucleotide sequence. The putative signal peptide sequence is underlined; four conserved cysteine residues that define the C-type lectin domain are shaded and underlined. The EPS motif for ligand binding specificity is in box. The WND motif is in double lines. The mutation position is in red. (B) Comparison of a putative CRD of Aalb_CTL1 with known and putative mosquito C-type lectins. Alignment of the CRD domain from Aalb_CTL1, Aaeg CTL16 [VectorBase: AAEL000533-RA], Aaeg CTL20 [VectorBase: AAEL011407-RA], Aaeg CTL25 [VectorBase: AAEL000556-RA], Aaeg mosGCTL-1 [VectorBase: AAEL000563-RA], Aalb_CTL2 [Genbank: AAV90641], Cq CTL54 [VectorBase: CPIJ014105], Agam CTL4 [VectorBase: ENSANGG00000018677], Agam CTLMA2 [VectorBase: ENSANGG00000018421]. Invariant or highly conserved residues within the CRD [Drickamer, 1993] are shown in the upper row: the italic singleletter amino acid codes indicate invariant conserved amino acids. Other abbreviations: $\Theta$, aliphatic; $\Phi$, aromatic, $\Omega$ aliphatic or aromatic; $\mathrm{O}$, oxygen-containing; Z, Glutamine or glutamic acid; triangle: the position for mutation. Cysteine residues forming disulphide bonds are marked with asterisks. Numbers on the left indicate amino acid positions starting from the initial methionine. 
that calcium was important for Aalb_CTL1 to possess hemagglutinating activity [25]. In addition to WND motif, an imperfect EPS motif $\left(\mathrm{Glu}^{128}-\mathrm{Pro}^{129}-\mathrm{Ser}^{130}\right)$ was also present in the CRD of Aalb_CTL1. For a C-type lectin of uncharacterized function, it can be predicted that CRDs containing a WND motif and an EPN motif might be involved in carbohydrate-binding, whereas, the specificity for either mannose or galactose depends on whether the motif is EPN or QPD [25]. Therefore, it could be further postulated that Aalb_CTL1 might be mannose-specific though the EPN was substituted by EPS in Aalb_CTL1.

Blast analysis with the deduced amino acid sequence of Aalb_CTL1 revealed that Aalb_CTL1 shared a high degree of similarity with several known or putative Ctype lectin family members from mosquitoes, especially the CTL from Ae. aegypti (Aaeg CTL16, similarity 83\%).

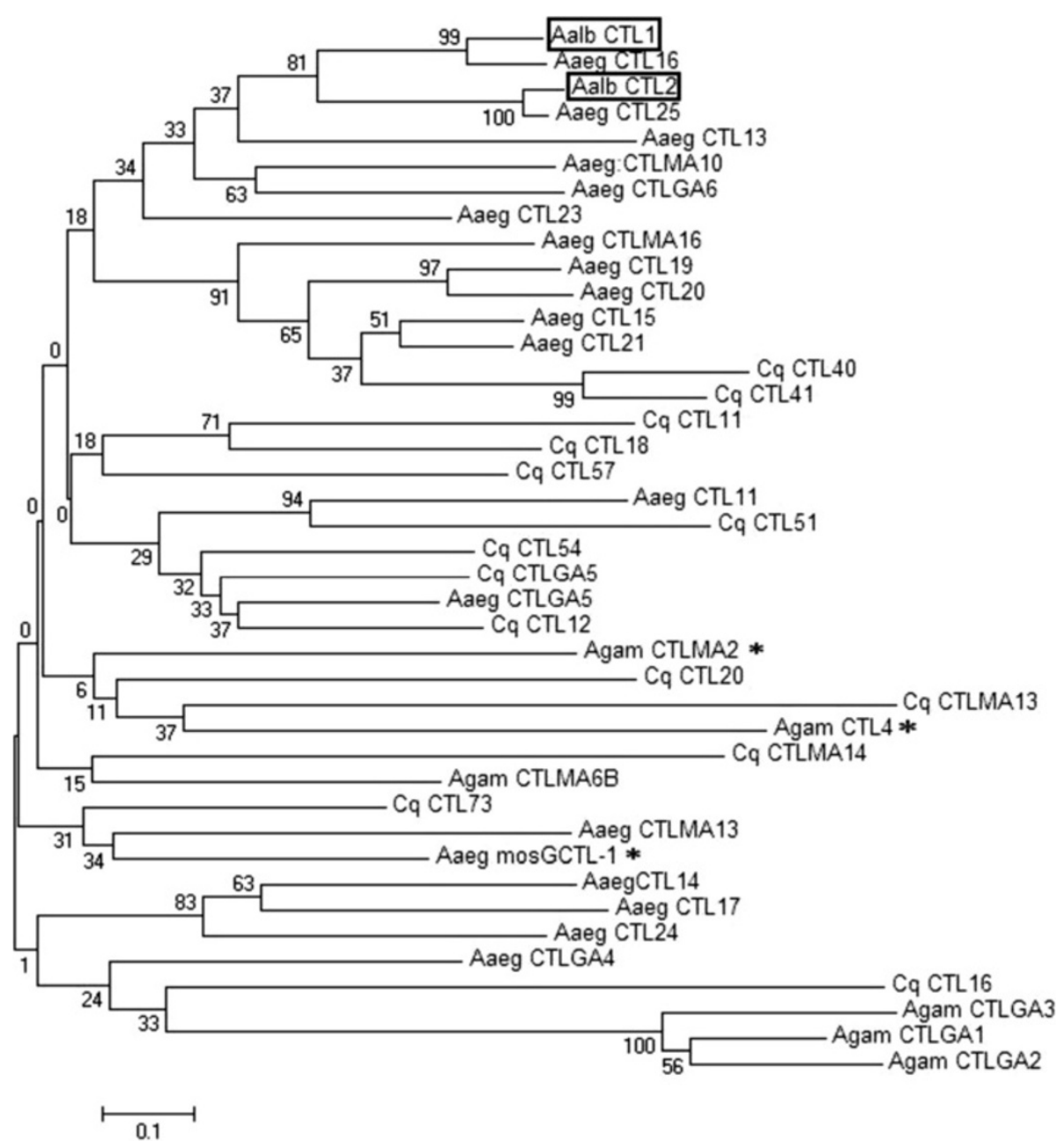

Figure 2 Phylogenetic tree constructed based on full-length amino acids sequence of Aalb_CTL1 and putative or known C-type lectins from mosquitoes. The C-type lectin sequences were from Aaeg CTL11 [VectorBase: AAEL008299], Aaeg CTL13 [VectorBase: AAEL004679], Aaeg CTLGA4 [VectorBase: N41092], Aaeg CTL14 [VectorBase: AAEL011453], Aaeg CTLGA5 [VectorBase: AAEL005641-RA], Aaeg CTLMA10 [VectorBase: AAEL011079-RA], Aaeg CTL15 [VectorBase: AAEL012353-RA], Aaeg CTLGA6 [VectorBase: AAEL009209], Aaeg CTL17 [VectorBase: AAEL011446-RA], Aaeg CTL19 [VectorBase: AAEL011404-RA], Aaeg CTLMA13 [VectorBase: AAEL011621-RA], Aaeg CTL20 [VectorBase: AAEL011407-RA], Aaeg CTL21 [VectorBase: AAEL011408-RA], Aaeg CTL23 [VectorBase: AAEL006456-RA], Aaeg CTL24 [VectorBase: AAEL002524-RA], Aaeg CTL25 [VectorBase: AAEL000556-RA], Aaeg mosGCTL-1 [VectorBase: AAEL000563-RA], Aaeg CTLMA16 [VectorBase: AAEL000283-RA], Aaeg CTL16 [VectorBase: AAEL000533-RA], Cq_CTL11[VectorBase: CPIJ000449], Cq_CTL12 [VectorBase: CPIJ001323], Cq_CTL16 [VectorBase: CPIJ003650], Cq_CTL18 [VectorBase: CPIJ004339], Cq_CTL20 [VectorBase: CPIJ004916], Cq_CTLMA13 [VectorBase: CPIJ007062], Cq_CTL40 [VectorBase: CPIJ007868], Cq_CTL41 [VectorBase: CPIJ007869], Cq_CTL51 [VectorBase: CPIJ012307], Cq_CTL54 [VectorBase: CPIJ014105], Cq_CTL57 [VectorBase: CPIJ015095], Cq_CTLMA14 [VectorBase: CPIJ015742], Cq_CTL73 [VectorBase: CPIJ016688], Cq_CTLGA5 [VectorBase: CPIJ017075], Agam_CTLMA6B [VectorBase: ENSANGG00000018449], Agam_CTLMA2 [VectorBase: ENSANGG00000018421], Agam_CTL4 [VectorBase: ENSANGG00000018677], Agam_CTLGA1 [VectorBase: ENSANGG00000009790], Agam_CTLGA2 [VectorBase: ENSANGG000000017954], Agam_CTLGA3 [VectorBase: ENSANGG00000009745]. In the Phylogenetic analysis, Aalb_CTL1 and Aalb_CTL2 are in the box from Ae. albopictus. Agam CTLMA2, Agam CTL4 and Aaeg mosGCTL-1 whose function has been reported and are marked with*, The bar (0.1) indicates genetic distance. 



Figure 3 Temporal and spatial expression profiles of Aalb_CTL1 $(\mathbf{n}=\mathbf{6})$. (A) tissue expression profile of Aalb_CTL1 in Ae. albopictus. SG: salivary gland, MG: midgut, FB: fat body. (B) stage-dependent expression of Aalb_CTL1 after blood-feeding. BSG_0: 0 time after engorgement; BSG_24: 24 hours after engorgement; BSG_72 hours after engorgement.

These mosquito CTLs contain only one CRD (Figure 1B), which is different from most of the CTLs from the Lepidopteran containing dual CRDs [26]. In order to understand the evolutionary relationship of Aalb_CTL1 in mosquitoes, a phylogenetic tree was constructed with the CTLs sequences from Ae. aegypti, An. gambiae and $C x$. quinquefasciatus that was highly similar with Aalb_ CTL1. In the phylogenetic tree (Figure 2), the Aalb_ CTL1 was clustered with Aaeg CTL16 firstly, then clustered with Aalb_CTL2 and Aaeg CTL25, and they further formed a big branch with some CTLs from Culex subfamily. CTLs from Anopheles subfamily were clustered together with the other CTLs from Culex subfamily. The relationships displayed in the phylogenetic tree showed that Aalb_CTL1 could belong to Culexspecific proteins. The above-mentioned evidences in our data has supported the Batholomay's notion [27] that Aaeg CTL16 locates in the upper part of the phylogenetic tree and no CTLs from An. gambiae and Drosophila melanogaster are clustered together with it.

\section{Tissue-specific expression and stage-dependent expression of Aalb_CTL1}

The tissue-specific expression and temporal expression pattern of Aalb_CTL1 in the salivary gland after bloodfeeding were determined by Real-Time qRT-PCR. The expression of Aalb_CTL1 at transcription level was much higher in the salivary gland than those in the midgut and fat body (Figure 3A, $\mathrm{P}<0.001$ ), indicating that Aalb_CTL1 would be expressed specifically in salivary gland and could be one of the important components in saliva as a secreted protein. Aaeg CTL16 from Ae. aegypti, revealing a high degree of sequence identity with Aalb_CTL1, has been verified to be salivary glandspecific lectin by semi-quantitative RT-PCR [27]. The expression level of Aalb_CTL1 was significantly downregulated at 0 time after blood-feeding (Figure 3B,
$\mathrm{P}<0.05)$, suggesting that the expression of Aalb_CTL1 transcript could be modulated by blood-feeding.

\section{Hemagglutinating activity ( $\mathrm{HA})$ and sugar binding specificity of rAalb_CTL1}

It is well known that not all proteins containing C-type CRDs can actually bind carbohydrate or even calcium [25]. To test the biological function of Aalb_CTL1, a recombinant plasmid pET-28a-Aalb_CTL1 was transformed

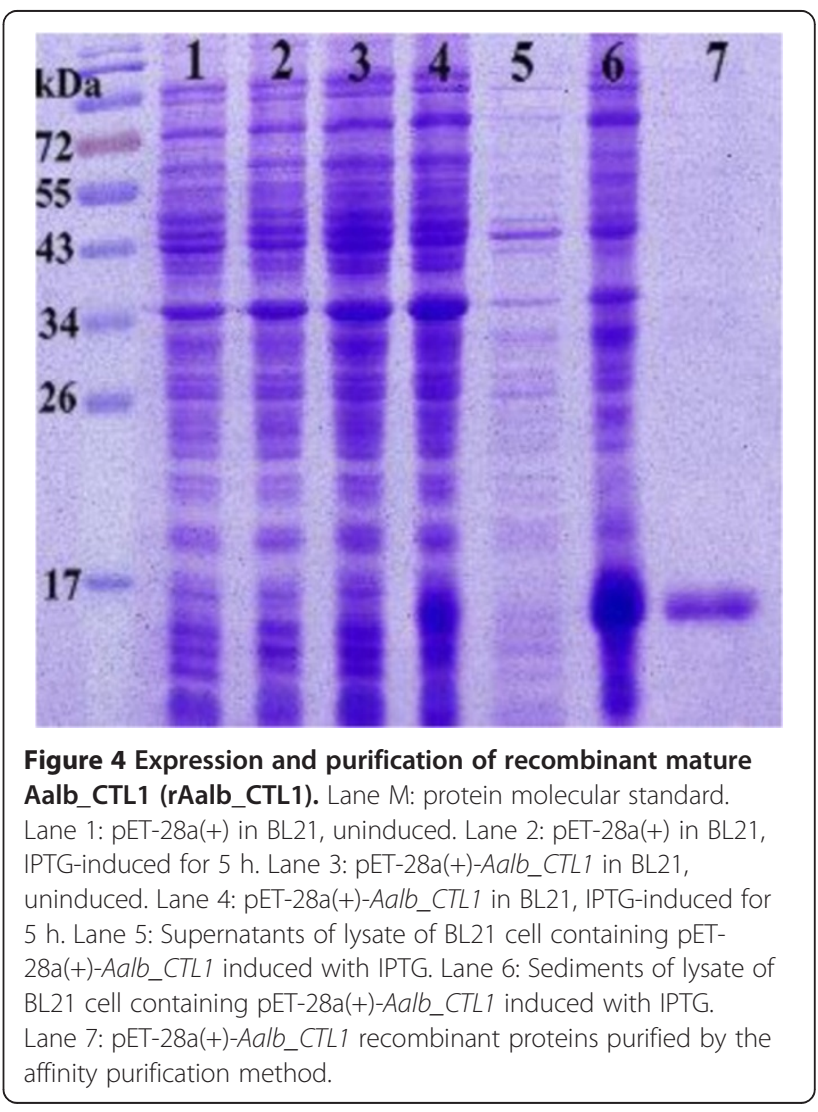


Table 2 Sugar binding specificities of rAalb_CTL1

\begin{tabular}{ll}
\hline Sugar name & Minimal Inhibitory Concentration (MIC) (mM) \\
\hline D-Glucose & 25 \\
D-Mannose & 6.25 \\
D-Galactose & 25 \\
Lactose & 50 \\
Sucrose & 50 \\
Maltose & 25 \\
\hline
\end{tabular}

and expressed in E. coli BL21 (DE3). One clear band of the rAalb_CTL1 with molecular mass of $17 \mathrm{kDa}$ was detected by SDS-PAGE analysis (Figure 4). The hemagglutination assay showed that rAalb_CTL1 at $40 \mathrm{mM} \mathrm{CaCl}{ }_{2}$ could induce the hemaggutination of rabbit erythrocytes, rat erythrocytes and mouse erythrocytes, and no agglutination was observed in the rAalb_CTL1 without $\mathrm{CaCl}_{2}$ group and BSA-Ca negative control under the same conditions. Furthermore, the agglutination was inhibited in the presence of EDTA, indicating that the agglutination of animal erythrocytes by rAalb_CTL1 was $\mathrm{Ca}^{2+}$-dependent. The agglutinating activity of rAalb_CTL1 for rabbit erythrocytes was higher than that for mouse and rat erythrocytes at a minimal agglutination concentration of $6.25 \mu \mathrm{g} / \mathrm{ml}$, compared with the minimal agglutination concentration of $12.5 \mu \mathrm{g} / \mathrm{ml}$ for mouse erythrocytes and rat erythrocytes.

The carbohydrate-binding inhibitory hemagglutination assay demonstrated that the hemagglutinating activity of rAalb_CTL1 were inhibited by all the six types of sugars at different concentrations in a calcium-dependent way (Table 2), but among the carbohydrates tested, Dmannose inhibited the hemagglutinating activity of rAalb_CTL1 most effectively with a minimal concentration of $6.25 \mathrm{mM}$. The results suggested that rAalb_CTL1 might have a high ligand affinity to bind mannose, which was associated with their structural features.

\section{Agglutination of rAalb_CTL1 to microbes}

CTLs are pattern recognition receptors that can distinguish self from non-self in mammalians [28]. Many studies on insects demonstrated that CTLs were involved in the insect innate immunity either in vivo or in vitro, especially in Lepidopterans $[29,30]$. In the present study,

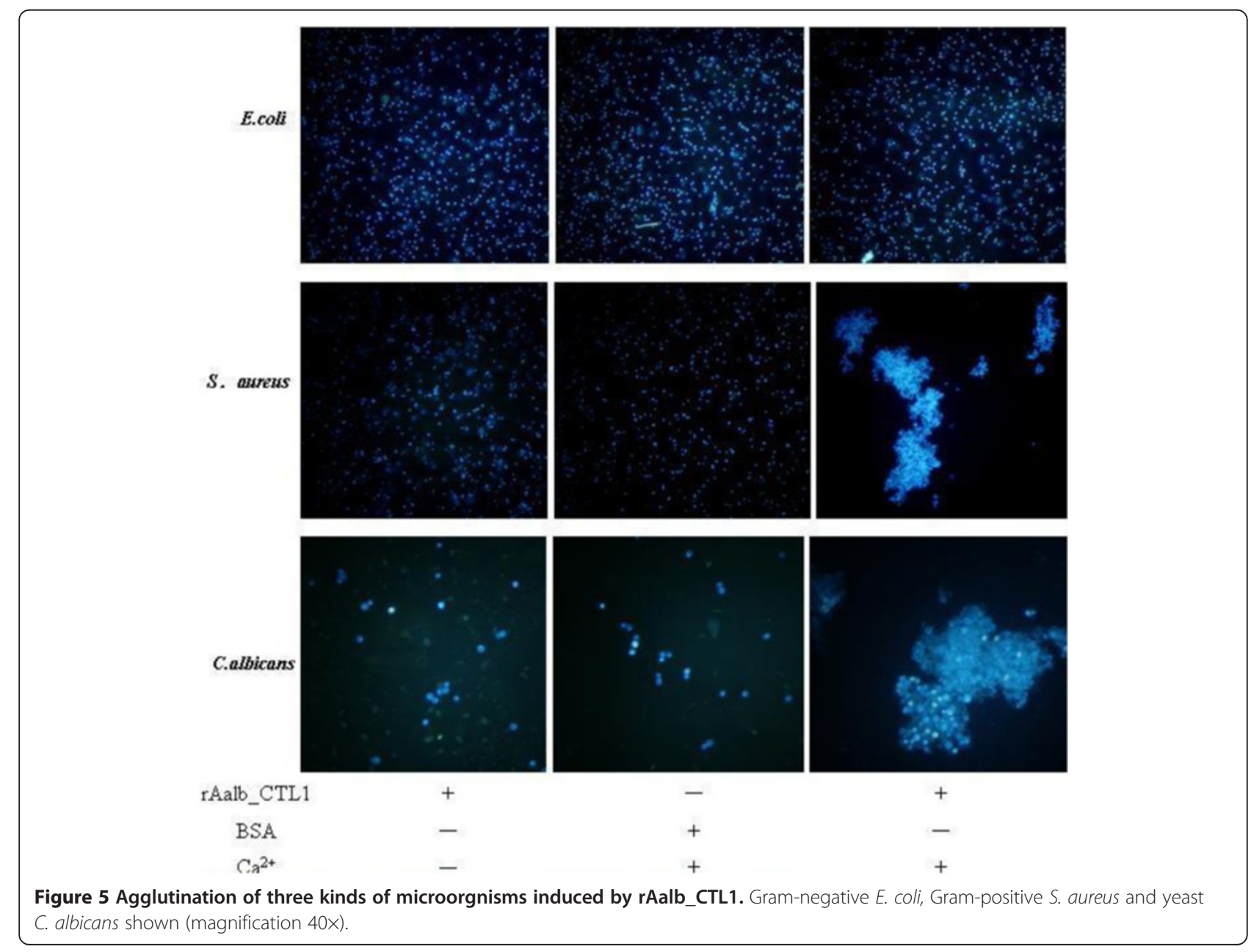


to determine whether rAalb_CTL1 could induce the aggregation of microbial pathogens, DAPI-labeled yeast C. albicans, Gram-positive bacteria $S$. aureus and Gram-negative bacteria $E$. coli were incubated with the rAalb_CTL1. The rAalb_CTL1 displayed the activity to agglutinate yeast $C$. albicans and Gram-positive bacteria $S$. aureus, but no agglutinating activity toward Gramnegative bacteria $E$. coli. Meanwhile, there was also no agglutinating activity observed in the BSA-Ca control and the rAalb_CTL1 without calcium group, exhibiting that the agglutinating activity against yeast $C$. albicans and Gram-positive bacterium $S$. aureus was in a calciumdependent fashion (Figure 5). The agglutinating activity of rAalb_CTL1 suggested that Aalb_CTL1 might be involved in eliminating pathogens from food. In the mosquito $A e$. aegypti, immunity-related proteins expressed in salivary gland, such as lysozymes and C-type lectins, are postulated to have a role in controlling bacterial growth in sugar solutions stored in the crop or in the gut following a blood meal $[21,31,32]$. In addition, a previous study on CTLs of An. gambiae showed that CTL4 and CTLMA2 can recognize the surface molecules of Gram-negative $E$. coli but not Gram-positive S. aureus, which is different from that of rAalb_CTL1. Furthermore, the defensive function against Gram-negative bacteria is attributed to the CTL4CTLMA2 heterodimer in vivo [16]. Thus, combined with the reasonable deduction of the result of the phylogenetic tree, it could be inferred that they might belong to the different members of CTLs and be involved in the recognition of different types of microorganisms.

\section{Conclusions}

In this study, a putative C-type lectin (Aalb-CTL1) was successfully cloned and expressed from Ae. albopictus Guangzhou strain. Aalb_CTL1 was only clustered with CTLs from Culex subfamily in the phylogenetic tree, suggesting that it would be a Culex-specific protein. Aalb_CTL1 was expressed specifically in salivary gland and its transcript level could be regulated by bloodfeeding. rAalb-CTL1 exhibited not only the hemagglutinating activity and a high affinity for mannose but also the agglutinating activity against yeast $C$. albicans and Gram-positive bacteria S. aureus in a calcium-dependent manner. Taken together, our results showed that Aalb_ CTL1 was a mannose-binding C-type lectin and could act as a PPR involved in the anti-microbial activity. More efforts should be focused on its role on the relationship of mosquito and pathogen in saliva in the future.

\footnotetext{
Abbreviations

Ae. albopictus: Aedes albopictus; Ae. aegypti: Aedes aegypti; An. gambiae: Anopheles gambiae; Cx. quinquefasciatus: Culex quinquefasciatus; E. coli: Escherichia coli; S. aureus: Staphylococcus aureus; C. albicans: Candida albicans; CTLs: C-type lectins; Aalb_CTL1: C-type lectin 1 in salivary gland of Ae. albopictus; CRD: Carbohydrate recognition domain; PRRs: Pattern recognition receptors; IPTG: Isopropyl $\beta$-D-Thiogalactoside; SDS-PAGE:
}

Sodium dodecyl sulfate polyacrylamide gel electrophoresis; BSA: Bovine serum albumin; Real-Time qRT-PCR: Real-Time quantitative reverse transcription polymerase chain reaction.

\section{Competing interests}

All the authors declare that they have no competing interests.

\section{Authors' contributions}

CJZ participated in the experiment design, carried out the experiment and drafted the manuscript. WY analyzed the data and drafted the manuscript. LFZ and LJ carried out bioinformatics analysis and prokaryotic expression. SY collected the tissue samples including salivary gland, midgut and fat body. WJH conceived of the study, analyzed the data and wrote the manuscript. All authors read and approved the final version of the manuscript.

\section{Acknowledgements}

The authors acknowledge Dr. Tongyan Zhao for her kindness in providing the Guangzhou strain of Ae. albopictus and Dr. Zhu Zeng for proofreading the revised version of manuscript. This research is also supported by the National Natural Science Foundation of China (No. 30600515 and No. 81060138).

\section{Author details}

'Department of Parasitology, Guiyang Medical College, Guiyang, Guizhou 550004, China. 'Lab for Modern Pathogen Biology, Guiyang Medical College, Guiyang, Guizhou 550004, China. ${ }^{3}$ Guizhou Center for Disease Control and Prevention, Guiyang, Guizhou 550004, China. ${ }^{4}$ Affiliated Hospital of Guiyang Medical College, Guiyang, Guizhou 550004, China.

Received: 28 January 2014 Accepted: 8 July 2014

Published: 22 July 2014

\section{References}

1. Janeway CA, Medzhitov R: Innate immune recognition. Annu Rev Immunol 2002, 20:197-216.

2. Sharon N, Lis H: Lectins: cell-agglutinating and sugar-specific proteins. Science 1972, 177:949-959.

3. Weis WI, Taylor ME, Drickamer K: The C-type lectin superfamily in the immune system. Immunol Rev 1998, 163:19-34.

4. Hoffmann JA, Kafatos FC, Janeway CA, Ezekowitz RA: Phylogenetic perspectives in innate immunity. Science 1999, 284:1313-1318.

5. Tan YY, Liu Y, Zhao XF, Wang JX: Characterization of a C-type lectin from the cotton bollworm, Helicoverpa armigera. Dev Comp Immunol 2009, 33:772-779

6. Koizumi N, Imamura M, Kadotani T, Yaoi K, Iwahana H, Sato R: The lipopolysaccharide-binding protein participating in haemocyte nodule formation in the silkworm Bombyx moriis is a novel member of the C-type lectin superfamily with two different tandem carbohydraterecognition domains. FEBS Lett 1999, 443:139-143.

7. Yu XQ, Kanost MR: Immulectin-2, a pattern recognition receptor that stimulates hemocyte encapsulation and melanization in the tobacco hornworm, Manduca sexta. Dev Comp Immunol 2004, 28:891-900.

8. Yu XQ, Kanost MR: Immulectin-2, a lipopolysaccaride-specific lectin from an insect, Manduca sexta, is induced in response to gram-negative bacteria. J Biol Chem 2000, 275:37373-37381.

9. Tan WB, Wang X, Cheng P, Liu LJ, Wang HF, Gong MQ, Quan X, Gao HG, Zhu CL: Molecular cloning and preliminary function study of iron responsive element binding protein 1 gene from cypermethrinresistant Culex pipens pallens. Parasit Vectors 2011, 4:215. doi:10.1186/ 1756-3305-4-215.

10. Holt RA, Subramanian GM, Halpern A, Sutton GG, Charlab R, Nusskern DR, Wincker P, Clark AG, Ribeiro JM, Wides R, Salzberg SL, Loftus B, Yandell M, Majoros WH, Rusch DB, Lai Z, Kraft CL, Abril JF, Anthouard V, Arensburger P, Atkinson PW, Baden H, de Berardinis V, Baldwin D, Benes V, Biedler J, Blass C, Bolanos R, Boscus D, Barnstead M, et al: The genome sequence of the malaria mosquito Anopheles gambiae. Science 2002, 298:129-149.

11. Nene V, Wortman JR, Lawson D, Haas B, Kodira C, Tu ZJ, Loftus B, Xi Z Megy K, Grabherr M, Ren Q, Zdobnov EM, Lobo NF, Campbell KS, Brown SE, Bonaldo MF, Zhu J, Sinkins SP, Hogenkamp DG, Amedeo P, Arensburger P, Atkinson PW, Bidwell S, Biedler J, Birney E, Bruggner RV, Costas J, Coy MR, 
Crabtree J, Crawford M, et al: Genome Sequence of Aedes aegypti, a major arbovirus vector. Science 2007, 316:1718-1723.

12. Arensburger $\mathrm{P}$, Megy $\mathrm{K}$, Waterhouse RM, Abrudan J, Amedeo P, Antelo B, Bartholomay L, Bidwell S, Caler E, Camara F, Campbell CL, Campbell KS, Casola C, Castro MT, Chandramouliswaran I, Chapman SB, Christley S, Costas J, Eisenstadt E, Feschotte C, Fraser-Liggett C, Guigo R, Haas B, Hammond M, Hansson BS, Hemingway J, Hill SR, Howarth C, Ignell R, Kennedy RC, et al: Sequencing of Culex quinquefasciatus establishes a platform for mosquito comparative genomics. Science 2010, 30:86-88.

13. Mike AO, George KC, Fotis CK: Effects of mosquito genes on plasmodium development. Science 2004, 303:2030-2032.

14. Cheng G, Cox J, Wang P, Krishnan MN, Dai J, Qian F, Anderson JF, Fikrig E: A C-type lectin collaborates with a CD45 phosphatase homolog to facilitate West Nile virus infection of mosquitoes. Cell 2010, 142:714-725. doi:10.1016/j.cell.2010.07.038.

15. Liu Y, Zhang FC, Liu JY, Xiao XP, Zhang SY, Qin CF, Xiang Y, Wang PH, Cheng G: Transmission-blocking antibodies against mosquito C-type lectins for Dengue prevention. Plos Pathog 2014, 10:e1003931.

16. Schnitger AK, Yassine H, Kafator FC, Osta MA: Two C-type lectins cooperate to defend Anopheles gambiae against Gram-negative bacteria. J Biol Chem 2009, 284:17616-17624.

17. Arcà B, Lombardo F, Francischetti IM, Pham VM, Mestres-Simon M, Andersen JF, Ribeiro JM: An insight into the sialome of the adult female mosquito Aedes albopictus. Insect Biochem Mol Biol 2007, 37:107-127.

18. Wu JH, Cheng JZ, Sun Y, Chen L: Selection of control genes in Real-time qPCR analysis of gene expression in Aedes albopictus. Chinese J Zoonoses 2011, 17:432-435 (in Chinese).

19. Luo T, Yang H, Li F, Zhang $X, X u X$ : Purification, characterization and cDNA cloning of a novel lipopolysaccharide-binding lectin from the shrimp Penaeus monodon. Dev Comp Immunol 2006, 30:607-617.

20. Champagne DE: Antihemostatic strategies of blood-feeding arthropods. Curr Drug Targets 2004, 4:375-396.

21. Ribeiro JM, Arca B, Lombardo F, Calvo E, Phan VM, Chandra PK, Wikel SK: An annotated catalogue of salivary gland transcripts in the adult female mosquito, Aedes aegypti. BMC Genomics 2007, 8(1):6. doi:10.1186/ 1471-2164-8-6.

22. Arca B, Lombardo F, Valenzuela JG, Francischetti IM, Marinotti O, Coluzzi M, Ribeiro JM: An updated catalogue of salivary gland transcripts in the adult female mosquito, Anopheles gambiae. J Exp Biol 2005, 208(Pt 20):3971-3986.

23. Drickamer $\mathrm{K}$ : Ca2+-dependent carbohydrate-recognition domains in animal proteins. Curr Opin Struct Biol 1993, 3:393-400.

24. Yamamoto-Kihara M, Kotani E: Isolation and characterization of a C-type lectin CDNA specifically expressed in the tip of mouthparts of the flesh fly Sarcophaga peregrine. Insect Mol Biol 2004, 13:133-140.

25. Zelensky AN, Gready JE: The C-type lectin-like domain superfamily. FEBS J 2005, 272:6179-6217.

26. Wang JL, Liu XS, Zhang Q, Zhao HB, Wang YF: Expression profiles of six novel C-type lectins in response to bacterial and $20 \mathrm{E}$ injection in the cotton bollworm (Helicoverpa armigera). Develop Comp Immuno 2012, 2:221-232.

27. Bartholomay LC, Waterhouse RM, Mayhew GF, Campbell CL, Michel K, Zou Z, Ramirez JL, Das S, Alvarez K, Arensburger P, Bryant B, Chapman SB, Dong YM, Erickson SM, Karunaratne SHPP, Kokoza V, Kodira CD, Pignatelli P, Shin SW, Vanlandingham DL, Atkinson PW, Birren B, Christophides GK, Clem RJ, Hemingway J, Higgs S, Megy K, Ranson H, Zdobnov EM, Raikhel AS, et al: Pathogenomics of Culex quinquefasciatus and Meta-Analysis of Infection Responses to Diverse Pathogens. Science 2010, 330:88-90.

28. Geijtenbeek TB, van Vliet SJ, Engering AT, Hart BA, van Kooyk Y: Self- and nonself-recognition by C-type lectins on dendritic cells. Annu Rev Immunol 2004, 22:33-54.

29. Kanost MR, Jiang H, Yu XQ: Innate immune responses of a Lepidopteran insect. Manduca sexta Immunol Rev 2004, 198:97-105.

30. Seufi AM, Galal FH, Hafez EE: Characterization of Multisugar-Binding C-Type Lectin (SpliLec) from a Bacterial-Challenged Cotton Leafworm,
Spodoptera littoralis. PLOS ONE 2012, 7:e42795. doi:10.1371/journal. pone.0042795.

31. Rossignol PA, Lueders AM: Bacteriolytic factor in the salivary glands of Aedes aegypti. Comp Biochem Physiol B 1986, 83:819-822.

32. Marinotti O, James AA, Ribeiro JMC: Diet and salivation in female Aedes aegypti mosquitoes. J Insect Physiol 1990, 36:545-548.

doi:10.1186/1756-3305-7-337

Cite this article as: Cheng et al: Cloning and characterization of a mannose binding C-type lectin gene from salivary gland of Aedes albopictus. Parasites \& Vectors 2014 7:337.

\section{Submit your next manuscript to BioMed Central and take full advantage of:}

- Convenient online submission

- Thorough peer review

- No space constraints or color figure charges

- Immediate publication on acceptance

- Inclusion in PubMed, CAS, Scopus and Google Scholar

- Research which is freely available for redistribution

Submit your manuscript at www.biomedcentral.com/submit
C BioMed Central 